\title{
THE LIFE-CYCLE OF LEISHMANIA IN THE SANDFLY WITH SPECIAL REFERENCE TO THE FORM INFECTIVE TO THE VERTEBRATE HOST
}

\author{
R. KILLICK-KENDRICK
}

\section{SUMMARY}

A review is given of recent advances in studies on the life-cycles of leishmaniae in phlebotomine sandflies including observations on the morphology and attachment of the parasites, putative genetic exchange in Leishmania, newly found factors affecting the deve- lopment of the parasite in the fly, and the characteristics of metacyclic promastigotes related to their preadaptation for life in the vertebrate host.

\section{RÉsumé : Cycle évolutif de Leishmania chez le phlébotome. Mise au point sur la forme infestante pour l'hôte vertébré.}

L'auteur fait une mise au point sur les progrès récents obtenus dans l'étude du cycle évolutif de leishmanies chez le phlébotome en particulier sur la morphologie et l'attachement du parasite dans le tube digestif, les facteurs récemment découverts modifiant le développement intra-vectoriel du parasite, les échanges génétiques putatifs chez Leishmania, et les caractéristiques des formes promastigotes métacycliques en rapport avec leur pré-adaptation à la survie chez l'hôte vertébré.

\section{INTRODUCTION}

Advances in the colonisation of sandflies over the past twenty years led to new findings on the life-cycles of leishmaniae in the sandfly many of which have been summarized by Killick-Kendrick (1979) and Molyneux and KillickKendrick (1987). In the present paper, a brief review is given of recent advances with special reference to the form of promastigote now believed to be preadaptated for life in the vertebrate host (the so-called metacyclic promastigote).

\section{MORPHOLOGY OF Leishmania IN THE SANDFLY}

Confirmation that at least some amastigotes ingested by sandflies divide one or more times before transforming into promastigotes comes from a study on the life-cycle of L. $i$. chagasi in Lu. longipalpis (Lainson et al., 1987). Dividing amastigotes were seen in stained smears of the infected midguts of sandflies which had fed through a membrane on suspensions of amastigotes 24,36 and $43 \mathrm{~h}$ before dissection. It may be that a mammalian bloodmeal contains

Medical Research Council External Scientific Staff, Department of Biology, Imperial College at Silwood Park, Ascot, Berks SL5 7PY, England. a transformation blocking factor (Brun et al., 1976), and that the change from amastigote to promastigote is inhibited until the factor is degraded by the digestive enzymes of the sandfly.

Promastigotes of varying morphology are the predominant form in the thoracic and abdominal midguts and the stomodaeal valve. Killick-Kendrick et al. (1974) adopted the terms nectomonad and haptomonad to describe two forms of promastigotes of $L$. amazonensis seen by the electron microscope in the midguts of experimentally infected Lu. longipalpis. They defined nectomonads as long, slender, electron-dense unattached promastigotes and haptomonads as broad, electron-lucid promastigotes with modified flagella capable of producing electron-dense plaques (hemidesmosomes) inside the sheath, associated with attachment to the cuticular intima of the stomodaeal valve. The same terms have been used for similar forms seen in other infections (Warburg et al., 1986; Lawyer et al., 1987; Walters et al., 1987, 1989a, b). The definitions of nectomonads and haptomonads were challenged by Walters et al. (1989a) who preferred them simply to denote either free-swimming or attached promastigotes with no consideration of morphology. A separation into nectomonads and haptomonads was not seen in preliminary observations on the ultrastructure of L. i. infantum in P. ariasi (Killick-Kendrick et al., 1977c). Haptomonads seen by Lawyer et al. and Walters et al. were sometimes in division, whereas divi- 
ding haptomonads of L. amazonensis were not seen in Lu. longipalpis (Killick-Kendrick et al., 1974; Molyneux et al., 1975).

Division of promastigotes in sandflies is principally by binary fission. The sequence of events in the division of L. i. infantum in P. ariasi and L. $i$. chagasi in Lu. longipalpis (both natural pairs of parasite and vector) is $(i)$ the production of a daughter flagellum, (ii) division of the nucleus into two, (iii) division of the kinetoplast into two and (iv) separation into two individuals by longitudinal fission beginning at the anterior end (Molyneux and KillickKendrick, 1987; Walters et al., 1989b).

In the cuticular-lined parts of the foregut other than the mouthparts (oesophagus, pharynx and cibarium), the commonest morphological form is the paramastigote which differs from the promastigote by having the kinetoplast beside the nucleus. Its shape is oval or round and it is much less motile than the promastigote. Like haptomonads on the stomodaeal valve, paramastigotes attach to the cuticular intima of the foregut of the fly by the tip of the flagellum within which hemidesmosomes are formed (Killick-Kendrick et al., 1977a, 1988; Walters, 1989a, b)). Whereas Killick-Kendrick (1979) believed paramastigotes in the foregut did not divide, Walters et al. (1989b) interpreted their electronmicrographs of some paramastigotes of $L$. $i$. chagasi in the foregut of Lu. longipalpis as dividing forms. An equally plausible interpretation is that a parasite or a flagellum had been cut across a fold and a similar interpretation is possible with electronmicrographs of paramastigotes of $L$. panamensis in the foregut of Lu. gomezi believed by Walters et al. (1989a) also to be in division.

Paramastigotes were the principal morphological form of L. braziliensis recognised in electronmicrographs of the pylorus and ileum of experimentally infected $L u$. longipalpis (Killick-Kendrick et al., 1977b). Unlike paramastigotes of other species in the foregut of the sandfly, the hindgut forms of $L$. braziliensis were commonly in division and attachment, by hemidesmosomes, was at any part of the flagellum, not only the tip. Similar studies by Walters et al. (1989a) on another species of the Neotropical subgenus Viannia Lainson and Shaw, 1987, L. panamensis, in $L u$. gomezi, a sandfly assumed to be a natural vector (see Killick-Kendrick, 1990), showed that the predominant form attached to the intima of the hindgut was not a paramastigote, but a «spatulate-shaped promastigote ". This form was described for the first time in the gut of the sandfly. Paramastigotes were « comparatively scarce » and there was little evidence of proliferation of promastigotes in the hindgut, particularly in the pylorus and ileum. Other unusual features were the presence of nectomonads attached by hemidesmosomes (which is as a contradiction) and a heavy infection of the whole of the hindgut, from pylorus to anus, with the greatest densities of parasites in the pylorus, ileum and rectum; the last site was not previously considered to be colonised by species of Viannia.

The differences in the observations by Walters et al. (1989a) and other workers show that further studies of the ultrastructure of parasites of this subgenus in proven vectors are needed before the life-cycles of species of the subgenus Viannia can be fully compared with those of the suprapylarian parasites.

\section{Attachment of Leishmaniae IN THE SANDFly}

With one exception, all accounts of the ultrastructure of leishmaniae in sandflies have shown attachment of promastigotes to the microvilli of the abdominal midgut by the insertion of the flagella of the parasites (Killick-Kendrick et al., 1974; Kaddu \& Mutinga, 1981; Molyneux et al., 1986; Lawyer et al., 1987; Walters et al., 1987, 1989b). The exception is the only report, referred to above, on the ultrastructure of a species of Viannia, L. panamensis, in the midgut of a sandfly by Walters et al. (1989a) who suggested that, because the parasites moved quickly from the hindgut to the anterior of the thoracic midgut, they perhaps had no « need to stabilize themselves in the midgut by association with the microvilli ».

Warburg et al. (1989) devised an in vitro assay to investigate attachment of L. major and L. panamensis to the midgut epithelium of $P$. papatasi. Both parasites exhibited a much stronger affinity for the internal surface of the midgut than to other sandfly tissues. Tests with 13 sugars to see if the attachment was lectin-mediated gave negative or inconsistent results. A monoclonal antibody to a protein which occurs on the tip and localized patches along the length of leishmanial flagella substantially inhibited adhesion. The authors concluded that the recognition of the microvilli was probably mediated biochemically and that the recognition of cuticle was probably by a different means.

All attachment to the cuticular intima of both the hind and foreguts is mediated by hemidesmosomes. Similarly, if parasites invade the diverticulum, attached forms again display hemidesmosomes in the flagellar sheath (Molyneux et al., 1986). The molecular mechanism of the attachment is not known.

\section{Metacyclic PROMASTigotes}

Perhaps the greatest recent advance in studies of leishmaniae in the sandfly is the confirmation of the production of so-called metacyclic promastigotes, forms in the sandfly which appear to be already adapted for life in the vertebrate host (Sacks and Perkins, 1984; see reviews by Sacks, 1988, 1989). Stages of Leishmania assumed to be the stage infective to the vertebrate were seen in the mouthparts of sandflies many years ago and gave rise to Adler's 
assumption, now contested, that transmission by bite required that such parasites were in the proboscis. The general view now prevailing is that, while transmission could readily take place when parasites are in the mouthparts, regurgitation of infective promastigotes from more posterior parts is no less important (Killick-Kendrick, 1986; Warburg \& Schlein, 1986; Jefferies et al., 1986). Nevertheless, promastigotes in the proboscis are not uncommon having been recorded in more than twenty combinations of parasite and fly.

Striking features separating metacyclic forms from others in the sandfly are high motility, lack of attachment to sandfly tissues, the small size of the body and the presence of a long free flagellum with a length about twice that of the body. Measurements of 50 randomly encountered metacyclic promastigotes of L. major in a stained smear made from the proboscis of an experimentally infected specimen of $P$. papatasi revealed that they were monomorphic with a mean body length of $10.1 \pm$ SD $0.89 \mu \mathrm{m}$ and flagellum length of $20.5 \pm \mathrm{SD} 2.16 \mu \mathrm{m}$. The posterior end was sharply pointed and the flagellar pocket was relatively deep (1.5-2.5 $\mu \mathrm{m})$ (Killick-Kendrick, 1986). It has been suggested that paramastigotes of $L$. $i$. chagasi and $L$. panamensis might also be infective to the vertebrate host because of similarities in the ultrastructure of paramastigotes and amastigotes (Walters, 1989a, $b$ ). This evidence may not be considered enough in the absence of observations, discussed below, showing modifications to the surface membrane and changes in enzyme activity which are, apparently, related to an ability to resist complement and the respiratory burst of macrophages.

Sacks and Perkins (1985) sequentially assayed the infectivity of a standard number of promastigotes of $L$. major harvested from the guts of $P$. papatasi, and of L. mexicana from Lu. longipalpis, and showed a progressive increase with time. This suggests that metacyclic promastigotes are produced in increasing numbers as the infection develops in the alimentary tract of the sandfly.

With L. amazonensis in Lu. flaviscutellata and L. i. chagasi in Lu. longipalpis, Lainson et al. (1987) showed that at least some parasites were infective to hamsters $15 \mathrm{~h}$ after an infective bloodmeal. In studies on the morphology of L. i. chagasi in Lu. longipalpis, Lainson and Shaw (1988) concluded that from 15-120 h after an infective bloodmeal there were two lines of parasite development. One was the transformation of amastigotes directly into metacyclic promastigotes which then divided producing more metacyclic promastigotes. The other began with large, vacuolated amastigotes which, having divided at least twice, gave rise to large promastigotes which did not themselves divide and produced no obvious product. Lainson and Shaw's observations may explain the increase in infectivity with time reported by Sacks and Perkins (1985). The role of the " unproductive " line remains unexplained, nor is it known how long the non-dividing promastigotes can live, but
Lainson and Shaw (1988) suggested they might play a role in creating suitable conditions for the line of metacyclic promastigotes. The report by Lainson and Shaw is the only one of division of the forms infective to the vertebrate. According to Mallinson and Coombs (1989a) metacyclic promastigotes of $L$. major do not divide in vitro but they can change to multiplicative promastigotes over a period of 24-48 h. However, the behaviour of metacyclic promastigotes in culture may not be the same as in the midgut of the sandfly.

Evidence that metacyclic promastigotes are adapted for life in a vertebrate host comes from studies on cultured promastigotes. A major step was the finding that differences in the surface membrane of infective and non-infective forms could be exploited with lectins to separate the two forms (Sacks \& Perkins, 1984, 1985; Sacks et al., 1985; see also Ready \& Smith, 1988). The surface carbohydrates of logphase promastigotes differ from those of metacyclic promastigotes which are themselves broadly similar to those of amastigotes (Sacks et al., 1985; Saraiva et al., 1986). Sacks and Da Silva (1987) showed that the loss of agglutination to peanut agglutinin by metacyclic promastigotes of L. major was caused by the loss of expression of a glycolipid of the surface membrane of log-phase promastigotes. The different glycolipid of metacyclic promastigotes was a well-defined marker of these forms. Pimenta et al. (1990) then showed that the change from log-phase to metacyclic promastigotes was concomitant with the acquisition of novel carbohydrates which are associated with developmental modifications of lipophosphoglycan, the major surface glycoconjugate of these cells implicated in attachment to and survival in macrophages (see review by Turco, 1990). In studies on the ultrastructure of metacyclic promastigotes, Mallinson and Coombs (1989a) noticed an irregular, finely grained surface coat on metacyclic promastigotes of L. major. By a technique known as fracture-flip (Anderson Forsman \& Da Silva, 1988), Pimenta et al. (1989) showed with the electron microscope that the surface of metacyclic promastigotes differs from that of log-phase parasites by the presence of a network of fusiform filaments $44 \mathrm{~nm}$ in length which, they thought, was probably the developmental lipophosphoglycan unique to the metacyclic forms. The same workers examined the two forms of promastigotes by transmission electron microscope and showed that metacyclic promastigotes of $L$. major have a glycocalyx of $17 \mathrm{~nm}$ thickness whereas that of log-phase parasites was only $7 \mathrm{~nm}$.

From the time metacyclic promastigotes are deposited in the skin of the vertebrate host until they gain access to a macrophage, they are presumably exposed to the potentially lethal effects of complement which is, incidentally, exploited in gaining entry into the host cell (Da Silva et al., 1989). It is surprising, therefore, that tests of serum resistance of log-phase and metacyclic promastigotes of different species have given contradictory results (refs in Mal- 
linson \& Coombs, 1989b). The possible influence of sandfly saliva on the survival of metacyclic promastigotes in the skin has not, however, been taken into account in these observations. Titus and Ribeiro (1988) described a striking enhancement of infectivity when minute amounts of sandfly saliva were added to inocula of cultured promastigotes of L. major and in vitro tests of complement sensitivity of metacyclic promastigotes should perhaps be done in the presence of saliva.

Mallinson and Coombs (1986) showed different levels of activity of a number of enzymes of infective and logphase promastigotes and commented that the changes in metacyclic forms correlated with enzyme activities of amastigotes suggesting that the infective parasites were preadapted at a molecular level to enter and survive in macrophages. Mallinson and Coombs (1989a) confirmed these results and also reported that the content of some amino acids of metacyclic promastigotes of $L$. major and $L$. mexicana was more similar to that of amastigotes than of midlog phase promastigotes. Furthermore, the endogenous and simulated respiration of the log-phase stages were appreciably higher than those of infective forms.

Mallinson and Coombs (1989b) studied the respiratory burst of macrophages after infection by promastigotes and found that, although this potentially lethal mechanism was not inhibited by metacyclic promastigotes (of L. major), the survival of the parasites was apparently good. Possibly these forms are adapted to resist the consequences of, rather than interfere with, the mechanism.

All the differences between infective and non-infective forms of promastigotes are obviously controlled genetically. Searle et al. (1989) reported observations on a gene which showed differential expression between infective and noninfective promastigotes and, by differentially screening a cDNA library constructed from stage-specific mRNA, Coulson and Smith (1990) isolated four clones encoding mRNAs which show elevated or unique expression in the metacyclic promastigotes of L. major. The same workers showed that there was no genomic rearrangement associated with changes in the morphology of promastigotes seen as infectivity to the vertebrate host is acquired.

\section{FACTORS AFFECTING THE LIFE-CYCLE}

\section{OF LEISHMANIAE IN THE SANDFLY}

Differences in the innate ability of different species of sandflies to support the growth of various species of Leishmania are well documented (refs in Killick-Kendrick, 1985). Schlein and Romano (1986) and Borovsky and Schlein (1987) studied the effect of $L$. major and $L$. donovani on the digestive enzymes of $P$. papatasi, a sandfly which supports a flourishing development of $L$. major but has an extremely low susceptibility to $L$. donovani. They showed that $L$. major depresses the enzyme activity but that
L. donovani enhances it, and suggested that low levels enabled $L$. major to thrive but high levels inhibited the growth of L. donovani. Earlier, Schlein et al. (1983) looked at the effect of turkey blood on the development of L. major in $P$. papatasi and found that the nucleated erythrocytes of the bird induced a high level of DNAase which was inimical to the parasite.

In recent work in which lines of $P$. papatasi susceptible and refractory to $L$. major were selected, variations in the susceptibility to Leishmania major by individual sandflies of the same species ( $P$. papatasi) have been shown to be genetically controlled (Wu \& Tesh, 1990b). The results of reciprocal crosses and crosses to the parent line suggested that neither character is dominant over the other and that different genes control susceptibility and refractoriness (Wu \& Tesh, 1990a). A completely refractory line was not obtained, perhaps because this trait is controlled by more than one gene. From the infection rates of $F_{1}$ crosses of the two lines, it was concluded that the observations could not be explained by maternal inheritance. At the time of writing, no information on the way in which the genes act has been reported.

The gut of sandflies is normally sterile and the development of leishmaniae is generally inhibited by bacteria and fungi. Warburg and Ostrovska (1987) described a similar effect caused by a cytoplasmic polyhedrosis virus which, although not invading the parasite, caused such severe structural and functional aberrations in the gut that it made it unsuitable for the parasite.

\section{Genetic EXChange In Leishmania}

Possible genetic exchange in Leishmania should be viewed against the backcloth of observations on the related genus Trypanosoma which is the more intensively studied of the two. The first firm evidence of genetic exchange in Trypanosoma came from the analysis of isoenzyme variations in stocks of T. brucei (Gibson et al., 1980; Tait, 1980). Jenni et al. (1986) then cloned recombinant forms of $T$. brucei from mice which had been infected with metacyclic forms of $T$. brucei from tsetse flies given meals of two genetically defined clones. This was confirmed by other workers and, from crosses of four different pairs of strains of $T$. brucei, a total of 61 hybrid clones has now been isolated. Although both meiosis and syngamy are involved, the order remains unknown. It is believed that exchange is not obligatory (Tait and Turner, 1990).

The first evidence of genetic exchange in Leishmania also came from isoenzyme studies in which patterns were seen, firstly by Maazoun et al. (1981) and then by Le Blanq et al. (1986), which suggested hybridization. Two stocks of Leishmania out of 132 isolated from man, dogs, gerbils and sandflies in $\mathrm{Al}$ Ahsa, Saudi Arabia, were later found with isoenzyme profiles looking like hybrids of L. major 
and L. arabica (Evans et al., 1989). Karyotyping and studies with kDNA probes supported this conclusion (Evans et al., 1987), although preliminary attempts to produce hybrids by cloning parasites from the midguts of $P$. papatasi which had been fed on mixtures of the two parasites met with no success (Evans et al., 1989). Similarly, Gradoni et al. (1986) were unable to find hybrids in P. perniciosus which had been infected by two distinct zymodemes of L. infantum.

In spite of the difficulty of producing hybrids experimentally, a recent report by Lanotte and Rioux (1990) will doubtless encourage further attempts. These workers videotaped cultured promastigotes of, firstly, L. infantum and then of L. tropica apparently fusing into a single individual. No details of the nuclei or kinetoplasts were visible in the living, unstained parasites, but it seems probable that conjugation between individuals of the same species was actually witnessed. No similar observations have been reported with trypanosomes.

\section{REFERENCES}

Anderson Forsman C., Da Silva P. P. : Fracture-flip: new highresolution images of cell surfaces after carbon stabilization of freeze-fractured membranes. J. Cell. Sci., 1988, 90, 531-541.

Borovsky D., Schlein Y.: Trypsin and chymotrypsin-like enzymes of the sandfly Phlebotomus papatasi infected with Leishmania and their possible role in vector competence. Med. Vet. Ent., 1987, 1, 235-242.

Brun R., Berens R. L., Krassner S. M. : Inhibition of Leishmania donovani transformation by hamster spleen homogenates and active human lymphocytes. Nature, 1976, 262, 689-691.

Coulson R. M. R., Smith D. F. : Isolation of genes showing increased or unique expression in the infective promastigotes of Leishmania major. Mol. Biochem. Parasitol., 1990 (in press).

Da Silva R. P., Fenton Hall B., Joiner K. A., Sacks D. L. : $\mathrm{CR} 1$, the $\mathrm{C} 3 \mathrm{~b}$ receptor, mediates binding of infecting Leishmania major metacyclic promastigotes to human macrophages. $J$. Immunol., 1989, 143, 617-622.

Evans D. A., Kennedy W. P. K., Elbihari S., Chapman C. J., Smith V., Peters W. : Hybrid formation with the genus Leishmania? Parassitologia, 1987, 29, 165-173.

Evans D. A., Smith V., Killick-Kendrick R., Neal R., Peters W. : Evidence for hybrid formation in the genus Leishmania. In: Leishmaniasis: the current status and new strategies for control (ed. D. T. Hart), Plenum, New York, 1989, 175-178.

Gibson W. C., Marshal T. F. de C., Godfrey D. G. : Numerical analysis of enzyme polymorphism. Adv. Parasitol., 1980, 18, 175-245.

Gradoni L., Gramiccia M., Maroli M., Pozio E. : Infestations mixtes de Phlebotomus perniciosus Newstead, 1911 par différents zymodèmes du complexe Leishmania infantum. In: Leishmania. Coll. int. CNRS/INSERM, 1986, 305-310.

Jefferies D., Livesey J. L., Molyneux D. H. : Fluid mechanics of bloodmeal uptake by Leishmania-infected sandflies. Acta Trop., 1986, 43, 43-53.

Jenni L., Marti S., Schweizer J., Betschart B., Le Page B. W. F., Wells J. M., Tait A., Paindavoine P., Pays E., Steinert M. : Hybrid formation between African trypanosomes during cyclical transmission. Nature, 1986, 322, 173-175.

Kaddu J. B., Mutinga M. J. : Leishmania in Kenyan phleboto- mine sandflies. 1. Leishmania aethiopica in the midgut of naturally infected Phlebotomus pedifer. Insect. Sci. Applic., 1981, 2, 245-250.

Killick-Kendrick R. : The biology of Leishmania in phlebotomine sandflies. In: Biology of Kinetoplastida, vol. II (eds W. H. R. Lumsden \& D. A. Evans), Academic Press, London/New York, 1979, 395-460.

Killick-Kendrick R. : Some epidemiological consequences of the evolutionary fit between leishmaniae and their phlebotomine vectors. Bull. Soc. Path. Exot., 1985, 78, 747-755.

Killick-Kendrick R. : The transmission of leishmaniasis by the bite of the sandfly. J. Roy. Army Med. Corps, 1986, 132, 134-140.

Killick-Kendrick R. : Phlebotomine vectors of the leishmaniases: a review. Med. Vet. Ent., 1990, 4, 1-24.

Killick-Kendrick R., Leaney A. J., Ready P. D., Molyneux D. H. : Leishmania in phlebotomid sandflies. IV. The transmission of Leishmania mexicana amazonensis to hamsters by the bite of experimentally infected Lutzomyia longipalpis. Proc. R. Soc. B, 1977a, 196, 105-115.

Killick-Kendrick R., Molyneux D. H., Ashford R. W. : Leishmania in phlebotomid sandflies. I. Modifications of the flagellum associated with attachment to the mid-gut and oesophageal valve of the sandfly. Proc. R. Soc. B, 1974, 187, 409-419.

Killick-Kendrick R., Molyneux D. H., Hommel M., Leaney A. J., Robertson E. S. : Leishmania in phlebotomid sandflies. V. The nature and significance of infections of the pylorus and ileum of the sandfly by leishmaniae of the braziliensis complex. Proc. Roy. Soc. B, 1977b, 198, 191-199.

Killick-Kendrick R., Molyneux D. H., Leaney A. J., Rioux J.A. : Aspects of the life-cycle of Leishmania in the sandfly. Proc. 2nd europ. Multicoll. Parasit., 1977 c, Trogir, 89-95.

Killick-Kendrick R., Wallbanks K. R., Molyneux D. H., Lavin D. P. : The ultrastructure of Leishmania major in the foregut and proboscis of Phlebotomus papatasi. Parasit. Res., 1988, 74, 586-590.

Lainson R., Ryan L., Shaw J. J. : Infective stages of Leishmania in the sandfly vector and some observations on the mechanism of transmission. Mem. Inst. Oswaldo Cruz, 1987, 82, 421-424.

Lainson R., Shaw J. J. : Observations on the development of Leishmania (L.) chagasi Cunha and Chagas in the midgut of the sandfly vector Lutzomyia longipalpis (Lutz and Neiva). Ann. Parasitol. Hum. Comp., 1988, 63, 134-145.

Lanotte G., Rioux J.-A. : Fusion cellulaire chez les Leishmania (Kinetoplastida, Trypanosomatidae). C. R. Acad. Sci. Paris, 1990, 310, 285-288.

Lawyer P. G., Young D. G., Butler J. F., Akin D. E. : Development of Leishmania mexicana in Lutzomyia diabolica and Lutzomyia shannoni (Diptera: Psychodidae). J. Med. Ent., 1987, 24, 347-355.

Le Blanq S. M., Schnur L. F., Peters W. : Leishmania of the Old World. I: The geographical and hostal distribution of L. major zymodemes. Trans. R. Soc. Trop. Med. Hyg., 1986, 80, 99-112.

Maazoun R., Lanotte G., Rioux J.-A., Pasteur N., KillickKendrick R., Pratlong F.: Signification du polymorphisme enzymatique chez les leishmanies. A propos de trois souches hétérozygotes de Leishmania infantum Nicolle, 1908, Leishmania cf. tarentolae Wenyon, 1921 et Leishmania aethiopica Bray, Ashford et Bray, 1973. Ann. Parasitol. Hum. Comp., 1981, 56, 467-475.

Mallinson D.J., Coombs G. H. : Molecular characterisation of the metacyclic forms of Leishmania. IRCS Med. Sci., 1986, 14, 557-558.

Mallinson D. J., Coombs G. H. : Biochemical characteristics of the metacyclic forms of Leishmania major and L. mexicana mexicana. Parasitology, 1989a, 98, 7-15. 
Mallinson D. J., Coombs G. H. : Interaction of Leishmania metacyclics with macrophages. Int. J. Parasitol., 1989b, 19, 647-656.

Molyneux D. H., Killick-Kendrick R. : Morphology, ultrastructure and life-cycles. In: The Leishmaniases in Biology and Medicine (eds W. Peters and R. Killick-Kendrick), Academic Press, London, 1987, 121-176.

Molyneux D. H., Killick-Kendrick R., Ashford R. W. : Leishmania in phlebotomid sandflies. III. The ultrastructrue of Leishmania mexicana amazonensis in Lutzomyia longipalpis. Proc. R. Soc. B, 1975, 190, 341-357.

Molyneux D. H., Ryan L., Lainson R., Shaw J. J. : The Leishmania-sandfly interface. In : Leishmania. Taxonomie et phylogenèse. Applications éco-épidémiologiques. Coll. int. CNRS, IMEE, Montpellier, 1986, 311-324.

Pimenta P. F., Da Silva R. P., Sacks D. L., Pinto Da Silva P. : Cell surface nanoanatomy of Leishmania major as revealed by fracture-flip. A surface meshwork of $44 \mathrm{~nm}$ fusiform filaments identifies infective developmental stage promastigotes. Europ. J. Cell Biol., 1989, 48, 180-190.

Ready P. D., Smith D. F. Peanut lectin agglutination and isolation of infective forms of Leishmania major. Trans. R. Soc. Trop. Med. Hyg., 1988, 82, 418.

Sacks D. L. : Developmental biology of Leishmania promastigotes. In: The Biology of Parasitism (eds. P. T. Englund \& A. Sher), A. R. Liss, New York, 1988, 93-103.

Sacks D. L. : Metacyclogenisis in Leishmania promastigotes. Exper. Parasitol., 1989, 69, 100-103.

Sacks D. L., Da Silva R. P. : The generation of infective stage Leishmania major promastigotes is associated with the cell-surface expression and release of a developmentally regulated glycolipid. J. Immunol., 1987, 139, 3099-3106.

Sacks D. L., Hieny S., Sher A. : Identification of cell surface carbohydrate and antigenic changes between noninfective and infective developmental stages of Leishmania major promastigotes. J. Immunol., 1985, 135, 564-567.

Sacks D. L., Perkins P. V. : Identification of an infective stage of Leishmania promastigotes. Science, 1984, 223, 1417-1419.

Sacks D. L., Perkins P. V. : Development of infective stage Leishmania promastigotes within phlebotomine sandflies. Am. J. Trop. Med. Hyg., 1985, 34, 456-459.

Saraiva E. M. B., Andrade A. F. R., Pereira M. E. A. : Cell surface carbohydrate of Leishmania mexicana amazonensis: differences between infective and non-infective forms. Europ. J. Cell Biol., 1986, 40, 219-225.

Schlein Y., Romano H. : Leishmania major and L. donovani: effects on proteolytic enzymes of Phlebotomus papatasi (Diptera, Psychodidae). Exp. Parasitol., 1986, 62m, 376-380.
Schlein Y., Warburg A., Schnur L. F., Shlomai J. : Vector compatibility of Phlebotomus papatasi dependent on differentially induced digestion. Acta Trop., 1983, 40, 65-70.

Searle S., Campos A. J. R., Coulson R. M. R., Spithill T. W., Smith D. F.: A family of heat shock protein 70-related genes are expressed in the promastigotes of Leishmania major. Nucl. Acids Res., 1989, 17, 5081-5095.

Tait A. ; Evidence for diploidy and mating in trypanosomes. Nature, 1980, 287, 536-538.

Tait A., Turner C. M. R. : Genetic exchange in Trypanosoma brucei. Parasitol. Today, 1990, 6, 70-75.

Titus R. G., Ribeiro J. M. C. : Salivary gland lysates from the sandfly Lutzomyia longipalpis enhance Leishmania infectivity. Science, 1988, 239, 1306-1308.

Turco S. J. : The leishmanial lipophosphoglycan: a multifunctional molecule. Exp. Parasitol., 1990, 70, 241-245.

Walters L. L., Chaplin G. L., Modi G. B., Tesh R. B. : Ultrastructural biology of Leishmania (Viannia) panamensis (= Leishmania braziliensis panamensis) in Lutzomyia gomezi (Diptera: Psychodidae): a natural host-parasite association. Am. J. Trop. Med. Hyg., 1989a, 40, 19-39.

Walters L. L., Modi G. B., Chaplin G. L., Tesh R. B. : Ultrastructural development of Leishmania chagasi in its vector, Lutzomyia longipalpis (Diptera: Psychodidae). Am. J. Trop. Med. Hyg., 1989b, 41, 295-317.

Walters L. L., Modi G. B., Tesh R. B., Burrage T. : Host-parasite relationship of Leishmania mexicana mexicana and Lutzomyia abonnenci (Diptera: Psychodidae). Am. J. Trop. Med. Hyg., 1987, 36, 294-314.

Warburg A., Hamada G. S., Schlein Y., Shire D. : Scanning electron microscopy of Leishmania major in Phlebotomus papatasi. Zeit. fur Parasit., 1986, 72, 423-431.

Warburg A., Ostrovska K. : Cytoplasmic polyhedrosis viruses in Phlebotomus papatasi inhibit development of Leishmania major. J. Parasitol., 1987, 73, 578-583.

Warburg A., Schlein Y. : The effect of post-bloodmeal nutrition of Phlebotomus papatasi on the transmission of Leishmania major. Am. J. Trop. Med. Hyg., 1986, 35, 926-930.

Warburg A., Tesh R. B., McMahon-Pratt D. : Studies on the attachment of Leishmania flagella to sandfly midgut epithelium. J. Protozool., 1989, 36, 613-617.

Wu W.-K., Tesh R. B. : Genetic factors controlling susceptibility to Leishmania major infection in the sandfly Phlebotomus papatasi. Am. J. Trop. Med. Hyg., 1990a, 42 (in press).

Wu W.-K., Tesh R. B. : Selection of Phlebotomus papatasi (Diptera: Psychodidae) lines susceptible and refractory to Leishmania major infection. Am. J. Trop. Med. Hyg., 1990b, 42 (in press). 